\title{
Osteoblastic Osteosarcoma
}

National Cancer Institute

\section{Source}

National Cancer Institute. Osteoblastic Osteosarcoma. NCI Thesaurus. Code C53953.

A conventional osteosarcoma characterized by the predominance of osteoid matrix. 